\title{
Galactic orbits of stars with planets ${ }^{\star}$
}

\author{
M. Barbieri ${ }^{1}$ and R. G. Gratton ${ }^{2}$ \\ 1 Dipartimento di Astronomia, Università di Padova, Vicolo dell'Osservatorio 2, 35122 Padova, Italy \\ e-mail: barmau@pd.astro.it \\ 2 Osservatorio Astronomico di Padova, Vicolo dell'Osservatorio 5, 35122 Padova, Italy \\ e-mail: gratton@pd.astro.it
}

Received 7 August 2001 / Accepted 5 December 2001

\begin{abstract}
We have reconstructed the galactic orbits of the parent stars of exoplanets. For comparison, we have recalculated the galactic orbits of stars from the Edvardsson et al. (1993) catalog. A comparison between the two samples indicates that stars with planets are not kinematically peculiar. At each perigalactic distance stars with planets have a metallicity systematically larger than the average for the comparison sample. We argue that this result favors scenarios where the presence of planets is the cause of the higher metallicity of stars with planets.
\end{abstract}

Key words. stars: planetary systems - stars: abundances

\section{Introduction}

Spectroscopic analysis of parent stars of exoplanets had shown that these stars are more metal-rich than field stars (Gonzalez 1996; Butler et al. 2000). Two scenarios had been proposed to explain the high metallicity: (i) During the build-up of planets, the gravitational interaction among them (or with the disk) injects some objects in high-eccentricity orbits that can intersect the surface of the host star. If a sufficient number of these objects are captured by the star, then the photospheric metallicity will be enhanced by the dissolution of the planet. (ii) Planet formation is enhanced by the high metallicity of the parent protostar nebula.

If there is a correlation between metal abundances and other properties of the stars that should have no impact on the formation of planets (e.g. their kinematics), we may expect systematic differences between stars with planets and without planets, in the first scenario, while there should be no difference in the second scenario.

In this paper we study the second hypothesis. Following the work by Allen \& Santillán (1991), using stellar parallaxes and proper motions from Hipparcos, we have reconstructed the galactic orbit of the parent stars of

Send offprint requests to: M. Barbieri,

e-mail: barmau@pd.astro.it

* Tables 1,2, and 4 are only available in electronic form at the CDS via anonymous ftp to

cdsarc.u-strasbg.fr (130.79.128.5) or via

http://cdsweb.u-strasbg.fr/cgi-bin/qcat?J/A+A/384/879 exoplanets. For comparison, we have recalculated the galactic orbit from the Edvardsson et al. (1993) catalogue, adopting a solar galactocentric distance of $8.5 \mathrm{kpc}$ instead of the $8.0 \mathrm{kpc}$ used by Edvardsson. A comparison between the two samples indicates that they are quite similar, and therefore that the stars with planets are not kinematically peculiar.

\section{The samples}

The stars with planets (SWP) are stars similar to the Sun; this peculiar characteristic is an intrinsic selection effect of discovery methods of radial velocities. The SWP sample we consider includes 58 stars, located within 70 pc of the Sun. Most of them are single; only a few stars are wide binaries, with a separation larger than $700 \mathrm{AU}$ between the two components. We have retrieved the list of SWP from the Extrasolar Planets Encyclopaedia at http://www.obspm.fr/encycl/encycl.html maintained by J. Schneider. The samples considered by the Geneva and Marcy and Butler groups (that have discovered the vast majority of extrasolar planets discovered so far, and carry most of the weight in our discussion) do not contain any kinematic bias. The samples considered by these two groups preferentially include low activity stars, excluding in this manner most of the stars with ages $<2$ Gyr.

The comparison sample is the same sample used by Edvardsson et al. (1993) to study the chemical evolution of the galactic disk. The sample of Edvardsson is composed of 189 nearby $(d<80 \mathrm{pc}) \mathrm{F}$ and $\mathrm{G}$ disk stars. 
The stars are selected from the Olsen (1988) catalogue, in the metal abundance range $-1.1<[\mathrm{Me} / \mathrm{H}]<0.3$; they are brighter than $V \simeq 8.3$. Only stars that have evolved off the Zero Age Main Sequence by more than 0.4 mag were considered. This ensures that their sample does not contain stars younger than $1.5 \mathrm{Gyr}$, and only very few younger than 2 Gyr (see their Table 11). In this respect, the selection criteria are very similar to those considered for SWP. Due to the selection criteria used by Edvardsson et al. in their sample is not representative of the distribution of stars in the solar neighborhood with metallicity because it over-represents low-metallicity stars with respect to solar-metallicity stars. However, the sample may be used to study the dynamical properties of stars in the solar neighborhood in a given abundance range because no kinematical selection criterion was adopted.

Nine stars are in common between the two samples: HD 6434, HD 9826, HD 19994, HD 89744, HD 95128, HD 114762, HD 143761, HD 169830, HD 217014.

\subsection{Kinematical data}

All the SWP (except one: BD-10 3166) have been observed by Hipparcos satellites. Using SIMBAD we have retrieved parallaxes and proper motion data from the Hipparcos catalog. The radial velocity are taken from the initial reports if available, we have taken the values from otherwise Barbier-Brossat \& Figon (2000), Eggen (1998) and Carney et al. (1994) catalogs. The majority of these stars are classified as "High Proper Motion stars". Kinematical data for the SWP are given in Table 1 (only available in electronic form at the CDS).

The Edvardsson catalog does not contain data about the proper motion of the stars: proper motions and the radial velocities for this second sample were retrieved using SIMBAD.

\subsection{Abundances of the stars}

Some papers have been dedicated to the spectroscopic analysis of SWP: Gonzalez (1998), Gonzalez \& Vanture (1998), Gonzalez (1999), Gonzalez et al. (1999), Gonzalez \& Laws (2000), Gonzalez et al. (2001), Santos et al. (2000), Naef et al. (2001). From these works we notice that on average SWP are more metal-rich than field stars. We have taken the metallicity $[\mathrm{Fe} / \mathrm{H}]$ from these papers. Data are unavailable for a few stars; in these cases they were taken from Marsakov \& Shevelev (1988) and Cayrel de Strobel et al. (2001).

The Edvardsson catalog contains $[\mathrm{Fe} / \mathrm{H}]$ values for the stars.

While there may be systematic offsets between these different determinations, we have not tried any correction to the original values. For the nine stars in common, $[\mathrm{Fe} / \mathrm{H}]$ from Edvardsson et al. are on average smaller by $0.07 \pm 0.02 \operatorname{dex}(\mathrm{rms}$ of $0.07 \mathrm{dex})$. Physical data for SWP are listed in Table 2 (only available in electronic form at the CDS).

\section{Calculation of orbits}

We transformed the proper motions into the corresponding galactocentric velocity components $\Pi, \Theta$, and $Z$, and corrected them for the Standard Solar Motion and the Motion of the Local Standard of Rest (LSR). For the adjustment of Standard Solar Motion we used a solar motion of $(U, V, W)=(+10.4,+14.8,+7.3) \mathrm{km} \mathrm{s}^{-1}$, according to Mihalas \& Routly (1968). The adopted procedure follows the method of Johnson \& Soderblom (1987); however we adopted a right-handed reference frame with the $x$-axis pointing toward the anticenter. The $y$-axis is along the direction of galactic rotation, and the $z$-axis is toward the North Galactic Pole.

\subsection{Galactic model of mass distribution}

The equations of motion have been integrated adopting the model for the Galactic gravitational potential and corresponding mass distribution by Allen \& Santillán (1991).

In this model, the mass distribution of the Galaxy is described as a three component system: a spherical central bulge, and a flattened disk, both of the MiyamotoNagai form, plus a massive spherical halo. The gravitational potential is fully analytical, continuous everywhere, and has continuous derivatives; its simple mathematical form lead to a rapid integration of the orbits with high numerical precision. The model provides accurate representation of the Galactic rotation curve $V_{\mathrm{C}}(R)$ and the force $F_{z}(z)$ perpendicular to the Galactic Plane. The values obtained for the Galactic rotation constants are $A=12.95 \mathrm{~km} \mathrm{~s}^{-1} \mathrm{kpc}^{-1}$ and $B=-12.93 \mathrm{~km} \mathrm{~s}^{-1} \mathrm{kpc}^{-1}$ which are in good agreement with observational data.

The expression for the potential of three components is:

$\phi_{\mathrm{B}}(r, z)=-\frac{G M_{\mathrm{B}}}{\sqrt{r^{2}+z^{2}+b_{\mathrm{B}}^{2}}}$

$$
\begin{aligned}
\phi_{\mathrm{D}}(r, z)= & -\frac{G M_{\mathrm{D}}}{\sqrt{r^{2}+\left(a_{\mathrm{D}}+\sqrt{z^{2}+b_{\mathrm{D}}^{2}}\right)^{2}}} \\
\phi_{\mathrm{H}}(r, z)= & -\frac{G M_{\mathrm{H}}}{\varrho} \cdot \frac{\left(\frac{\varrho}{a_{\mathrm{H}}}\right)^{2.02}}{1+\left(\frac{\varrho}{a_{\mathrm{H}}}\right)^{1.02}}-\frac{M_{\mathrm{H}}}{1.02 \cdot a_{\mathrm{H}}} \\
& \times\left[-\frac{1.02}{1+\left(\frac{\varrho}{a_{\mathrm{H}}}\right)^{1.02}}+\ln \left(1+\left(\frac{\varrho}{a_{\mathrm{H}}}\right)^{1.02}\right)\right]_{\mathrm{R}}^{100}
\end{aligned}
$$

where $\varrho=\sqrt{r^{2}+z^{2}}$.

Table 3 lists the values of the various constants for this model. The total mass of the model is $9.0 \times 10^{11} M_{\odot}$, and the Halo is truncated at $100 \mathrm{kpc}$.

\section{Galactic orbits}

To perform the numerical integration, we utilized the Burlish-Stoer method, directly applied to the second 
Table 3. Constants for the galactic model.

\begin{tabular}{lcrr}
\hline \multirow{2}{*}{ Galactocentric distance of Sun } & $R_{\odot}$ & 8.5 & $\mathrm{kpc}$ \\
Local circular velocity & $\Theta$ & 220 & $\mathrm{~km} \mathrm{~s}^{-1}$ \\
& & & \\
Bulge & $M_{\mathrm{B}}$ & $1.41 \times 10^{10}$ & $M_{\odot}$ \\
& $b_{\mathrm{B}}$ & 0.3873 & $\mathrm{kpc}$ \\
Disk & $M_{\mathrm{D}}$ & $8.56 \times 10^{10}$ & $M_{\odot}$ \\
& $a_{\mathrm{D}}$ & 5.3178 & $\mathrm{kpc}$ \\
Halo & $b_{\mathrm{D}}$ & 0.2500 & $\mathrm{kpc}$ \\
& $M_{\mathrm{H}}$ & $80.02 \times 10^{10}$ & $M_{\odot}$ \\
& $a_{\mathrm{H}}$ & 12.0 & $\mathrm{kpc}$ \\
\hline
\end{tabular}

order differential equations that describe the motion of a star. This numerical method allows to obtain a typical error in energy and in the $z$-component of the angular momentum of the star of, respectively, $\Delta E / E \approx 10^{-4}$ and $\Delta L_{z} / L_{z} \approx 10^{-9}$. Orbits were back integrated long enough to obtain significant values for the main orbital parameters.

\subsection{Stars with planets}

We have not computed orbits for two stars: BD-10 3166 because the parallax is not known; and HD 222582 because the value of the heliocentric radial velocity of this star is not available from the literature.

Galactocentric orbit parameters for the remaining 56 stars are given in Table 4 (only available in electronic form at the CDS), where $R_{\mathrm{p}}$ is the perigalacticon, $R_{\mathrm{a}}$ is the apogalacticon, $z_{\max }$ is the maximum height above the Galactic Plane, $e$ is the eccentricity defined as $\frac{R_{\mathrm{a}}-R_{\mathrm{p}}}{R_{\mathrm{a}}+R_{\mathrm{p}}}, E$ is the total energy, $L_{z}$ is the $z$-component of the angular momentum of the stars.

\subsection{Stars of Edvardsson catalog}

The Edvardsson catalog contains 189 stars, but we have computed the orbits for 185 stars; radial velocities are not available in the literature for the four remaining stars (HD 98553, HD 155358, HD 159703, HD 218504).

We have recalculated the galactic orbit of stars of the Edvardsson catalog, although the data about perigalacticon are present in this catalog, because Edvardsson assumed a solar galactocentric distance of $8.0 \mathrm{kpc}$ rather than the value of $8.5 \mathrm{kpc}$ adopted in this paper (Kerr \& Lynden-Bell 1986).

\section{Results}

To understand whether the high metallicity of SWP is the cause or the effect of the presence of planets, we

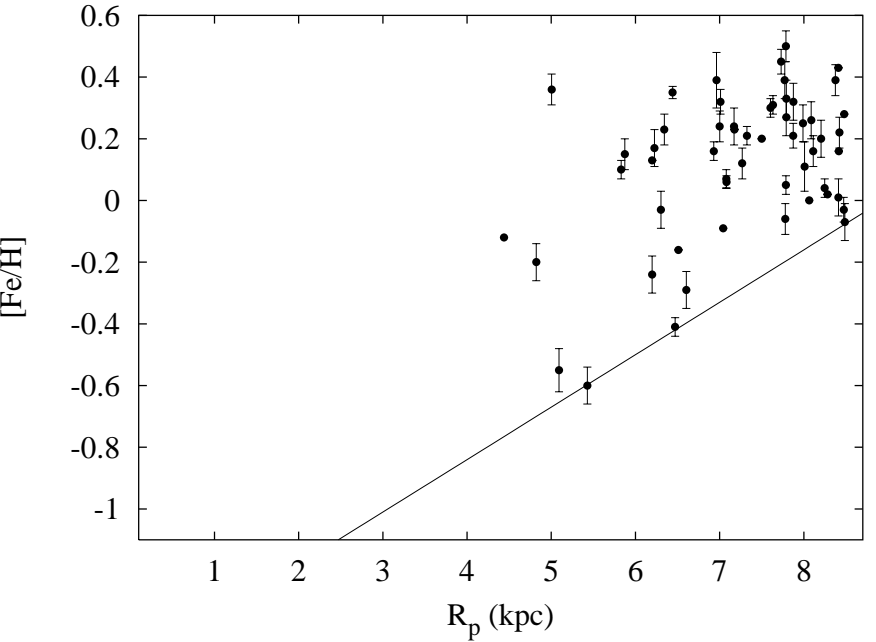

Fig. 1. Perigalactic distances for the SWP sample, the straight line represent the lower envelope of SWP distribution in this plane.

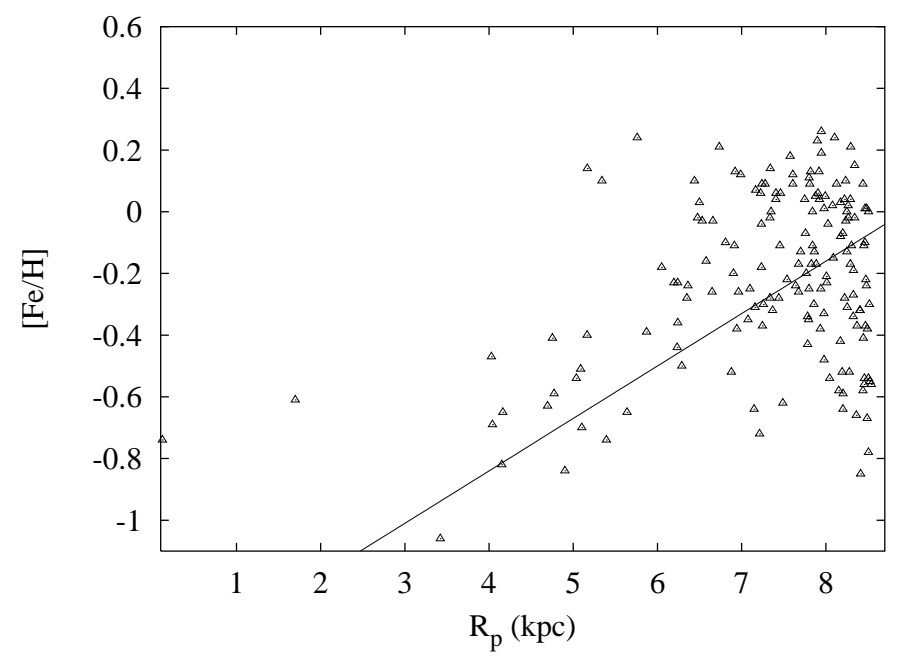

Fig. 2. Perigalactic distances for the Edvardsson sample; the straight line is the lower envelope of the SWP distribution.

plotted the iron abundance relative to perigalacticon for both samples.

Figures 1 and 2 give the $[\mathrm{Fe} / \mathrm{H}]$ versus perigalacticon for the two samples. These figures show that the distribution of metallicity versus perigalacticon of the two samples are quite similar: in both cases metallicity increases with perigalactic distance. To understand this trend (apparently opposite to the overall radial abundance gradient found for our Galaxy using various techniques), we note that our samples are local: stars with small perigalactic distances that presently are close to the Sun should be on highly eccentric orbits; they are drawn from the thick disk or old thin disk populations, and are on average much older (and metal-poor) than the stars on more circular orbits. Practically, our data indicates the presence of two different populations of SWP: an old population with perigalactic distance less than $6 \mathrm{kpc}$ with lower metallicity, 
and a young population with perigalactic distance more than $6 \mathrm{kpc}$ and with high metallicity.

\section{Discussion}

Our data may be used to discuss the metallicity-planet connection. In fact, if the high metallicity is the cause for the presence of planets, we should not expect any correlation between presence of planets and galactocentric distance: it is the overall metallicity that is important, and in a given metallicity bin the distribution of stars with perigalactic distance should be the same for stars with and without planets. On the other hand, if planet capture is the mechanism that enhances the metallicity, we should expect that the SWP are the upper envelope of the distribution of metallicity with galactocentric distance: at any galactocentric distance the stars with planets should be more metal-rich than average, and in a given metallicity bin, stars with planets should have on average smaller perigalactic distances.

At any galactocentric distance, the metallicity of the stars with planets is roughly the upper envelope of the metallicity distribution of the comparison sample. The few known (mildly) metal-poor stars with planets all have small values of the perigalactic distance, and there are no stars with known planets among the much more frequent (in the solar neighborhood) mildly metal-poor stars that have nearly circular orbits. Based on the relative frequency in the solar neighborhood of mildly metal-poor stars on circular and highly eccentric orbits, we should expect a large number of mildly metal-poor stars with planets. These are not observed.

A Kolmogorov-Smirnov test for the mildly metal-poor $([\mathrm{Fe} / \mathrm{H}] \leq-0.1)$ SWP and Edvardsson stars gives a probability that both distributions with perigalactic distances were extracted from the same parent population of 0.0046 ; the same test for the higher value of metallicity $([\mathrm{Fe} / \mathrm{H}]>-0.1)$ gives a probability of 0.5159 . This result does not depend critically on possible offsets between the abundance scales used for SWP and the Edvardsson sample: in fact, even if we lower $[\mathrm{Fe} / \mathrm{H}]$ for all SWP by $0.07 \mathrm{dex}$ (the systematic offset measured for the nine stars in common in the two samples), the possibility that the mildly metal-poor stars $([\mathrm{Fe} / \mathrm{H}] \leq-0.1)$ in the two samples were extracted from parent populations having the same distribution with perigalactic distance can still be rejected at a quite good level of confidence (the probability that they are drawn from the same distribution is 0.022 ). The test for the higher metallicity objects gives a probability of 0.6644 .

Note that this is not due to a selection effect related to the apparent brightness of the stars: in fact in the Edvardsson sample, metal-poor stars $([\mathrm{Fe} / \mathrm{H}] \leq-0.1)$ with $R_{\mathrm{p}} \leq 7 \mathrm{kpc}$ are on average fainter than metal-poor stars with $R_{\mathrm{p}}>7 \mathrm{kpc}$ (average magnitudes of $6.16 \pm 0.20$ and $5.80 \pm 0.12$ respectively). It should then be easier to discover planets around the brighter stars with near circular orbits than around the fainter stars with eccentric orbits.

In the scenario where high metallicity is the cause of the presence of planets, when the metallicity exceeds a critical value, the planets are present, independent of the perigalactic distance. Hence we expect that in a given metal abundance range, the number of stars with planets at different perigalactic distance will follow the distribution of the parent population of all nearby stars. We should then find more stars with planets with large perigalactic distance than with small perigalactic distance (since the parent population is more numerous).

In the opposite scenario, where the planets are the cause of the high metallicity of the central stars, the SWP are the upper envelope of the distribution of $[\mathrm{Fe} / \mathrm{H}]$ versus perigalactic distance of field stars: this is because at any perigalactic distance, stars with planets are metal enriched with respect to stars without planets. In this case we expect that in the mildly metal-poor abundance bin, we may find a larger number of stars with small perigalactic distance than stars with large perigalactic distance, because the metal enrichment caused by the presence of the planets will push the stars with planets extracted from the parent population of mildly metal-poor stars out of this metallicity bin (into the high metallicity bin).

We conclude that insofar as no kinematic selection effect is present in the sample of stars with planets, the fact that planets have been found only among mildly metalpoor stars (presently in the solar neighborhood) that have small perigalactic radii (in spite of the relative rarity of such objects) clearly favours the second scenario.

We argue that this result strongly favours scenarios where the presence of planets is the cause of the higher metallicities. Of course our results do not rule out the possibility that higher metallicity also favours the presence of planets (i.e. that both scenarios are applicable).

Acknowledgements. This research has made use of the SIMBAD data base, operated at CDS, Strasbourg, France. We thanks Dr. S. J. Aarseth for making available his code ORBIT, Dr. G. Carraro for useful discussion and suggestions, Dr. S. Desidera for a critical reading of the text, Dr. G. Marcy and Dr. M. Mayor for prompt responses to our inquiry about any possible kinematic bias in their sample, and the referee Dr. M. Crézé that helped to improv our paper.

\section{References}

Allen, C., \& Santillán, A. 1991, RMA\&A, 22, 255

Barbier-Brossat, M., \& Figon, P. 2000, A\&AS, 142, 217

Butler, R. P., Vogt, S., Marcy, G. W., et al. 2000, ApJ, 545, 504

Carney, B. W., Latham, D. W., Laird, J. B., \& Aguilar, L. A. 1994, AJ, 107, 2240

Cayrel de Strobel, G., Soubiran, C., \& Ralite, N. 2001, A\&A, 373,159

Edvardsson, B., Andersen, J., Gustafsson, B., et al. 1993, A\&A, 275,101

Eggen, O. J. 1998, AJ, 115, 2397 
Gonzalez, G. 1996, MNRAS, 285, 403

Gonzalez, G. 1998, A\&A, 334, 221

Gonzalez, G., \& Vanture, A. D. 1998, A\&A, 339, L29

Gonzalez, G. 1999, MNRAS, 308, 447

Gonzalez, G., Wallerstein, G., \& Saar, S. H. 1999, ApJ, 511, L111

Gonzalez, G., \& Laws, C. 2000, AJ, 119, 390

Gonzales, G., Laws, C., Tyagi, S., \& Reddy, B. E. 2001, AJ, 121,432

Johnson, D. R. H., \& Soderblom, D. R. 1987, AJ, 93, 864

Kerr, F. J., \& Lynden-Bell, D. 1986, MNRAS, 221, 1023
Laws, C., \& Gonzalez, G. 2001, ApJ, 553, 405

Marsakov, V. A., \& Shevelev, Y. G. 1988, BICDS, 35, 129

Mihalas, D., \& Routly, P. M. 1968, Galactic Astronomy (San Francisco: Freeman)

Naef, D., Lathan, D. W., Mayor, M., et al. 2001, A\&A, 375, 27 Olsen, E. H. 1988, A\&A, 189, 173

Santos, N. C., Israelian, G., \& Mayor, M. 2000, A\&A, 363, 228

Santos, N. C., Israelian, G., \& Mayor, M. 2001, A\&A, 373, 1091

Schneider, J. 2001, http://www.obspm.fr/encycl/encycl.html 